\title{
Onset of Darcy-Brinkman convection with a uniform internal heat source and vertical throughflow
}

\author{
N. Deepika ${ }^{\mathrm{a}}$, P.A.L. Narayana ${ }^{\mathrm{a}}$, Antony A. Hill ${ }^{\mathrm{b}}$ \\ ${ }^{a}$ Department of Mathematics, Indian Institute of Technology Hyderabad, Kandi, Medak, India, \\ 502285 \\ ${ }^{b}$ Department of Applied Sciences, University of the West of England, Bristol, BS16 1QY, UK
}

\begin{abstract}
The problem of thermal convection in a horizontal fluid-saturated porous layer is examined, where the flow is governed by the Brinkman extension of Darcy's law. A uniform internal heat source and vertical throughflow are also considered. The linear and nonlinear stability analyses are performed in order to determine the stability characteristics of the system. The linear and nonlinear thresholds give good agreement in the absence of vertical throughflow. However, it is shown that there are potential regions of sub-critical instabilities for increasing values of internal heat source parameter $Q$, Péclet number $P e$ and Darcy number $D a$.
\end{abstract}

Key words: porous medium, energy stability analysis, throughflow, internal heat source

\section{Introduction}

The flow and heat transfer in porous media has been a highly active area of research for the past few decades due to broad range of applications in engineering field as well as in the hydrology of ground water, food processing, geophysics, and petroleum reservoirs etc.

Horton and Rogers [1] and Lapwood [2] were first to study the flow and heat transfer in a porous media. These studies are based on the Darcy law, which neglects the effects of inertial forces and solid boundary. However, in the case of highly porous materials, where these effects are significant. The permeability $K$ in the Brinkman equation is such that the equation reduces to a form of the Navier-stokes equation as $K \rightarrow \infty$ and to the Darcy equation as $K \rightarrow 0$. Brinkman [3] calculated the viscous force exerted by fluid flow on a dense swarm of particles embedded in a porous medium which lead to the formation of the Darcy-Brinkman model. In Brinkman [3] the simultaneous effects of boundary and inertia are discarded. Tam [4] specified that when the spatial length scale is much higher than $\frac{1}{\alpha}$ (where $\alpha^{2}=\frac{\mu}{K \tilde{\mu}}: \mu$ is the viscosity, $\tilde{\mu}$ is the effective viscosity, $K$ is the permeability), the term $\Delta \mathbf{v}$ (linear in fluid flow $\mathbf{v}$ ) is insignificant in comparison with the linear term $\mathbf{v}$. In this case the Darcy-Brinkman model reduces to the Darcy 
law. Later, Vafai and Tien [5] analyzed the inertia and solid boundary effects on flow and heat transfer in porous media, in which the local volume averaging technique was used to establish the governing equations. These effects are more pronounced in high Prandtl-number fluids, high permeable porous media and for large pressure gradients. It is noted that the effect of Brinkman term will only be in thin layers adjacent to rigid boundary, mainly within a distance $K^{1 / 2}$. Nield [6] confirmed that Brinkman model is applicable to porous media whose porosities are greater than 0.6. Vafai and Kim [7] addressed the work of Nield [8] and conveyed that the effect of porosity variation is not required for high porous medium, but it should be considered for dense porous medium. Walker and Homsy [9] studied the convective instabilities in an isotropic porous media. Later, Rees [10] analyzed the work of Walker and Homsy [9] in detail, by performing an asymptotic analysis. Barletta et al. [11] studied the convective instability in highly permeable porous medium, taking viscous dissipation into account.

The buoyancy force generated by heating at the bottom plate or an internal heat generation is one of the main sources of research interest for the onset of convection in a fluid-saturated porous medium. The onset of convection in a porous medium with internal heat generation is investigated in Gasser and Kazimi [12], in which the critical internal and external Rayleigh numbers were obtained for both stabilizing and destabilizing boundary conditions. Later, the finite amplitude convection in a porous layer heated from within was studied by Tveitereid [13] by analyzing steady solutions in the form of hexagons and two-dimensional rolls. Then after, Royer and Flores [14] studied natural convection in a rectangular heterogeneous anisotropic porous medium with an internal heat generation and compared numerical results of the problem with the cases of homogeneous and heterogeneous isotropic porous media. Subsequently, Parthiban and Patil [15] studied the thermal instability problem in an anisotropic porous media with internal heat source subjected to inclined gradients and obtained numerical results by using Galerkin technique. Nouri-Borujerdi et al. [16] studied the effect of uniform internal heat source in a horizontal porous layer with local thermal non equilibrium model, and then Nouri-Borujerdi et al. [17] focused on the influence of Darcy number on the onset of convection in the presence of uniform internal heat source where the equation of motion is modeled by Brinkman law.

The study of throughflow is important as it gives the possibility to control the potential convective instabilities by adjusting the throughflow. Wooding [18] was the first to study the Rayleigh instability of convection in a porous medium with throughflow. Convective flow in a two dimensional porous channel was studied by Sutton [19] when there is a net flow of fluid through the channel. Later, Homsy and Sherwood [20] provided the stability results for the problem of thermal convection for the case of net fluid discharge through porous medium, and obtained the linear and energy bounds. These bounds coincide at low discharge rates. The effect of throughflow on convection with inclined temperature gradients was addressed by Nield [21] and Qiao and Kaloni [22]. The onset of convection with internal heat generation and net throughflow (strong and weak) for different hydrodynamic boundary conditions is examined in the article by Khalili and Shivakumara [23]. The stability of a saline boundary layer formed by throughflow near surface of the porous medium was studied in Van Duijn et al. [24] and Pieters and Schuttelaars [25]. The linear instability of boundary layer formed by vertical throughflow 
in horizontal porous medium with local thermal nonequilibrium was examined in Patil and Rees [26]. The studies on the the effect of vertical throughflow and internal heat source on the onset of convection in a layered porous medium were found in Kuznetsov and Nield [27], [28]. Later, the combined effects of throughflow and variable gravity on Hadley-Prats flow was studied by Deepika and Narayana [29]. The effect of vertical throughflow of a non-newtonian power-law fluid on the onset of convection in a horizontal fluid saturated porous layer was examined in Barletta and Storesletten [30]. In the absence of Brinkman term, the present problem is similar to the problem considered in Barletta et al. [31], in which viscous dissipation is considered as an internal heat source just as uniform internal heat source considered in this manuscript.

Owing to the important applications of the vertical throughflow and internal heat source in engineering applications, we analyze these combined effects in a fluid saturated Darcy-Brinkman porous layer. Section 2 deals with the governing equations and basic state solution, which is followed by the linear theory in Section 3 and the nonlinear theory via energy method in section 4. Numerical results of both the linear and nonlinear theories are discussed in Section 5. From the numerical results, we observed that the combination of vertical throughflow and internal heat source significantly effects the stability of the convection pattern. These observations are concluded in section 6 .

\section{Mathematical Formulation}

Let us consider a horizontal fluid-saturated porous layer $\Omega$ bounded by two planes separated at a distance $L>0$, such that $\Omega=\mathbb{R}^{2} \times(-L / 2, L / 2)$ where $O x \bar{y} z$ to be the Cartesian coordinate system with $\bar{x}, \bar{y}$ being the horizontal axes and $\bar{z}$ being the vertical axis. The Oberbeck-Boussinesq approximation is assumed to be valid throughout the domain i.e. the density $\rho$ is constant everywhere except in the body force term and it can be expressed as

$$
\rho=\rho_{0}\left(1-\beta\left(\bar{T}-\bar{T}_{0}\right)\right)
$$

where $\rho_{0}$ is the density at reference temperature $\bar{T}_{0}, \bar{T}$ is the temperature, and $\beta$ is the thermal expansion coefficient.

The set of governing equations for the flow and heat transfer in dimensional form are given by

$$
\begin{gathered}
\bar{\nabla} \cdot \overline{\mathbf{u}}=0, \\
\frac{\mu}{K} \overline{\mathbf{u}}=-\bar{\nabla} \bar{P}+\mu_{e} \bar{\triangle} \overline{\mathbf{u}}+\rho g \hat{\mathbf{k}}, \\
(\rho h)_{m} \frac{\partial \bar{T}}{\partial \bar{t}}+\left(\rho h_{p}\right)_{f}(\overline{\mathbf{u}} \cdot \bar{\nabla}) \bar{T}=k \bar{\triangle} \bar{T}+q,
\end{gathered}
$$

where $\overline{\mathbf{u}}=(\bar{u}, \bar{v}, \bar{w})$ is the seepage velocity vector, $\bar{\triangle}=\frac{\partial^{2}}{\partial \bar{x}^{2}}+\frac{\partial^{2}}{\partial \bar{y}^{2}}+\frac{\partial^{2}}{\partial \bar{z}^{2}}, \bar{P}$ is the pressure, $\hat{\mathbf{k}}=(0,0,1)$ and $g$ is the acceleration due to gravity. Furthermore, $\mu, \mu_{e}, K, \bar{t}, \rho$ and $k$ are dynamic viscosity, effective viscosity, permeability, time, reference density of fluid and thermal conductivity, respectively. The term $q>0$ in (1c) is a (constant) internal heat source, and

$$
\left(\rho_{0} h\right)_{m}=(1-\phi)\left(\rho_{0} h\right)_{s}+\phi\left(\rho_{0} h_{p}\right)_{f}
$$


where $h_{p}$ is the specific heat of the fluid and $h$ is the specific heat of the solid, with the subscripts $f, s$ and $m$ referring to the fluid, solid and porous components respectively. The walls are assumed to be permeable, with a throughflow velocity $\bar{w}_{0}$ in the vertical direction such that

$$
\begin{gathered}
(\bar{u}, \bar{v}, \bar{w})=\left(0,0, \bar{w}_{0}\right), \quad \bar{T}=\bar{T}_{1}, \text { at } \bar{z}=-\frac{L}{2}, \\
(\bar{u}, \bar{v}, \bar{w})=\left(0,0, \bar{w}_{0}\right), \quad \bar{T}=\bar{T}_{0}, \text { at } \bar{z}=\frac{L}{2} .
\end{gathered}
$$

We define the following dimensionless variables

$$
\begin{gathered}
\mathbf{x}=(x, y, z)=\frac{1}{L}(\bar{x}, \bar{y}, \bar{z})=\frac{\overline{\mathbf{x}}}{L}, \quad \mathbf{u}=(u, v, w)=\frac{L}{\alpha}(\bar{u}, \bar{v}, \bar{w}), \quad t=\frac{\alpha}{L^{2} A} \bar{t} \\
A=\frac{(\rho h)_{m}}{\left(\rho h_{p}\right)_{f}}, \quad \alpha=\frac{k}{\left(\rho h_{p}\right)_{f}}, \quad P=\frac{K}{\alpha \mu}\left(\bar{P}+\rho_{0} g \bar{z}\right), \quad T=\frac{\bar{T}-\bar{T}_{0}}{\bar{T}_{1}-\bar{T}_{0}},
\end{gathered}
$$

where $\alpha$ is the thermal diffusivity, $A$ is the ratio of volumetric heat capacity $(\rho h)_{m}$ of fluid saturated porous medium to the volumetric heat capacity $\left(\rho h_{p}\right)_{f}$ of fluid. By substituting the dimensionless variables into (1a)-(1d), the following non-dimensional governing equations are obtained:

$$
\begin{gathered}
\nabla \cdot \mathbf{u}=0, \\
\mathbf{u}=-\nabla P+D a \triangle \mathbf{u}+R a \hat{\mathbf{k}}, \\
\frac{\partial T}{\partial t}+(\mathbf{u} \cdot \nabla) T=\triangle T+Q, \\
(u, v, w)=(0,0, P e), T=1, \text { at } z=-\frac{1}{2}, \\
(u, v, w)=(0,0, P e), T=0, \text { at } z=\frac{1}{2} .
\end{gathered}
$$

In the above equations, the dimensionless parameters are

$$
\begin{gathered}
D a=\frac{\mu_{e}}{\mu} \frac{K}{L^{2}}, \quad R a=\frac{\rho_{0} \beta g\left(\bar{T}_{1}-\bar{T}_{0}\right) K L}{\mu \alpha}, \\
Q=\frac{L^{2}}{k\left(\bar{T}_{1}-\bar{T}_{0}\right)} q, \quad P e=\frac{L}{\alpha} \bar{w}_{0},
\end{gathered}
$$

where, $D a$ is the Darcy number, $R a$ is the Rayleigh number, $Q$ is the coefficient of internal heat source, and $P e$ is the Péclet number. The steady-state solution to the equations (3a)-(3d) is given by

$$
\begin{gathered}
\mathbf{u}_{B}=\left(u_{B}, v_{B}, w_{B}\right)=(0,0, P e) \\
T_{B}=\frac{1}{2}+\frac{\left(1+\frac{Q}{P e}\right)}{2 \sinh \left(\frac{P e}{2}\right)}\left(\cosh \left(\frac{P e}{2}\right)-e^{P e z}\right)+\frac{Q}{P e} z .
\end{gathered}
$$

When there is no throughflow in the vertical direction, i.e., $P e=0$, the basic state solution tends to

$$
\mathbf{u}_{B}=(0,0,0), \quad p_{B}=p(x, y, z), \quad T_{B}=-\frac{Q}{2} z^{2}-z+\left(\frac{Q}{8}+\frac{1}{2}\right) .
$$

To study the stability of the basic state, the following perturbations are added

$$
\mathbf{u}=\mathbf{u}_{B}+\mathbf{U}, \quad p=p_{B}+P, \quad T=T_{B}+\theta,
$$


where $\mathbf{U}=(U, V, W)$, to yield the governing perturbation equations

$$
\begin{gathered}
\nabla \cdot \mathbf{U}=0, \\
\mathbf{U}=-\nabla P+D a \triangle \mathbf{U}+R a \theta \hat{\mathbf{k}}, \\
\frac{\partial \theta}{\partial t}+\mathbf{U} \cdot \nabla T_{B}+\mathbf{u}_{B} \cdot \nabla \theta+\mathbf{U} \cdot \nabla \theta=\triangle \theta, \\
W=\theta=0 \quad \text { at } z= \pm \frac{1}{2} .
\end{gathered}
$$

Equation (8) indicates that there is zero perturbation on velocity and temperature at the boundary planes.

\section{$3 \quad$ Linear stability analysis}

To proceed with the linear instability analysis, products of the perturbations in (8b) and (8c) are discarded. Since the resulting system is linear and autonomous, we may seek solutions of the form

$$
(U, V, W, \theta, P)=(U(\mathbf{x}), V(\mathbf{x}), W(\mathbf{x}), \theta(\mathbf{x}), P(\mathbf{x})) e^{\sigma t}
$$

where $\sigma=\sigma_{r}+i \sigma_{i}$ is the exponential growth rate parameter. Before proceeding further, we demonstrate that the linear stability analysis for the present problem admits the principle of the exchange of stabilities, i.e., $\sigma$ is real.

Substituting (9) into (8c) yields

$$
\left(\triangle-\sigma-\left(\mathbf{u}_{B} \cdot \nabla\right)\right) \theta-\frac{d T_{B}}{d z} W=0 .
$$

Taking the third component of double curl of (8b) yields

$$
R a \triangle_{2} \theta=\triangle W-D a \triangle^{2} W
$$

where

$$
\triangle=\frac{\partial^{2}}{\partial x^{2}}+\frac{\partial^{2}}{\partial y^{2}}+\frac{\partial^{2}}{\partial z^{2}}, \quad \triangle_{2}=\frac{\partial^{2}}{\partial x^{2}}+\frac{\partial^{2}}{\partial y^{2}}, \quad \nabla=\frac{\partial}{\partial x} \hat{\mathbf{i}}+\frac{\partial}{\partial y} \hat{\mathbf{j}}+\frac{\partial}{\partial z} \hat{\mathbf{k}} .
$$

From (10) and (11) it follows that

$$
\left(\triangle-\sigma-\left(\mathbf{u}_{B} \cdot \nabla\right)\right)\left(\triangle W-D a \triangle^{2} W\right)-R a \frac{d T_{B}}{d z} \triangle_{2} W=0,
$$

which subsequently implies

$$
(\triangle-\sigma) \triangle W-D a(\triangle-\sigma) \triangle^{2} W-\left(\mathbf{u}_{B} \cdot \nabla\right) \triangle W+D a\left(\mathbf{u}_{B} \cdot \nabla\right) \triangle^{2} W-R a \frac{d T_{B}}{d z} \triangle_{2} W=0 .
$$

Let $W^{*}=W_{r}-i W_{i}$ and $\theta^{*}=\theta_{r}-i \theta_{i}$ be the complex conjugates of $W=W_{r}+i W_{i}$ and $\theta=\theta_{r}+i \theta_{i}$, respectively. Now, multiplying equation (12) with $W^{*}$ and integrating over $V$ (where $V$ is a periodicity cell), leads to

$$
\begin{aligned}
\| \Delta & W\left\|^{2}+\sigma\right\| \nabla W\left\|^{2}+D a\right\| \nabla \triangle W\left\|^{2}+\sigma D a\right\| \triangle W \|^{2}-R a \int_{V} \frac{d T_{B}}{d z}\left|\nabla_{2} W\right|^{2} d V \\
- & \int_{V}\left(\left(\mathbf{u}_{B} \cdot \nabla\right) \triangle W\right) W^{*} d V+D a \int_{V}\left(\left(\mathbf{u}_{B} \cdot \nabla\right) \triangle^{2} W\right) W^{*} d V=0
\end{aligned}
$$


where $\|$.$\| denote norm on L^{2}(V)$. It follows that $\int_{V} \frac{d T_{B}}{d z}\left|\nabla_{2} W\right|^{2} d V \in \mathbb{R}$ since $\frac{d T_{B}}{d z} \in \mathbb{R}$ and

$$
\begin{aligned}
-\int_{V}\left(\left(\mathbf{u}_{B} \cdot \nabla\right) \triangle W\right) W^{*} d V= & \int_{V}\left(\left(\mathbf{u}_{B} \cdot \nabla\right) \nabla W\right) \cdot \nabla W^{*} d V \\
= & \left\langle\left(\mathbf{u}_{B} \cdot \nabla\right) \nabla\left(W_{r}+i W_{i}\right), \nabla\left(W_{r}-i W_{i}\right)\right\rangle \\
= & \left\langle\left(\mathbf{u}_{B} \cdot \nabla\right)\left(\nabla W_{r}, \nabla W_{r}\right)\right\rangle-i\left\langle\left(\mathbf{u}_{B} \cdot \nabla\right)\left(\nabla W_{r}, \nabla W_{i}\right)\right\rangle \\
& +i\left\langle\left(\mathbf{u}_{B} \cdot \nabla\right)\left(\nabla W_{i}, \nabla W_{r}\right)\right\rangle+\left\langle\left(\mathbf{u}_{B} \cdot \nabla\right)\left(\nabla W_{i}, \nabla W_{i}\right)\right\rangle \\
& =\left\langle\left(\mathbf{u}_{B} \cdot \nabla\right)\left(\nabla W_{r}, \nabla W_{r}\right)\right\rangle+\left\langle\left(\mathbf{u}_{B} \cdot \nabla\right)\left(\nabla W_{i}, \nabla W_{i}\right)\right\rangle \in \mathbb{R} .
\end{aligned}
$$

Similarly $\int_{V}\left(\left(\mathbf{u}_{B} \cdot \nabla\right) \triangle^{2} W\right) W^{*} d V \in \mathbb{R}$. Now, taking the imaginary part of (13) gives

$$
\sigma_{i}\left(\|\nabla W\|^{2}+D a\|\triangle W\|^{2}\right)=0
$$

Therefore $\sigma_{i}=0$. Hence, the only existing modes are stationary modes in the linear theory.

Introducing the normal modes of the form

$$
W=W(z) f(x, y) \quad \theta=\theta(z) f(x, y)
$$

where $f(x, y)$ is plan-form which tiles the plane $(x, y)$ with $\triangle_{2} f=-a^{2} f$ such that $a^{2}=a_{x}{ }^{2}+a_{y}{ }^{2}$ ( $a_{x}$ is the wave number in $x$-direction, $a_{y}$ is the wave number in the $y$-direction, $a$ is the overall wave number). The plan-form indicates the horizontal shape of the convection cells formed at the onset of convection. These cells form a regular horizontal pattern tiling the $(x, y)$ plane, where the wave number $a$, is a measure of the width of the convection cell. By substituting normal modes (14) in (10) and (11), the obtained eigenvalue problem for linear instability is

$$
\begin{gathered}
D a\left(D^{2}-a^{2}\right)^{2} W-\left(D^{2}-a^{2}\right) W-a^{2} R a \theta=0, \\
\left(D^{2}-a^{2}\right) \theta-\frac{d T_{B}}{d z} W-P e D \theta=0 . \\
z= \pm \frac{1}{2}: \quad W=D W=\theta=0 .
\end{gathered}
$$

where $D=d / d z$. Numerical thresholds for linear instability generated by (15a)-(15c) are given in Section 5.

\section{Nonlinear stability analysis}

To study the nonlinear stability, we define the energy functional as follows

$$
E(t)=\frac{\xi}{2}\|\theta\|^{2},
$$

where $\xi$ is a positive coupling parameter. Multiplying equation (8b) by $\mathbf{U}$, equation (8c) by $\theta$, integrating over $V$ (where $V$ is a periodicity cell) and applying the divergence theorem, the following equations are obtained:

$$
\begin{gathered}
\|\mathbf{U}\|^{2}=-D a\|\nabla \mathbf{U}\|^{2}+R a\langle\theta, W\rangle, \\
\frac{1}{2} \frac{d}{d t}\|\theta\|^{2}+\left\langle\left(\mathbf{U} \cdot \nabla T_{B}\right), \theta\right\rangle=-\|\nabla \theta\|^{2},
\end{gathered}
$$


where $\|\cdot\|$ and $\langle\cdot, \cdot\rangle$ denote the norm and inner product on $L^{2}(V)$, respectively. Now, differentiating (16) with respect to $t$ and using equations (17)-(18), we get

$$
\frac{d E}{d t}=I-D
$$

where

$$
\begin{gathered}
I=-\xi\left\langle\left(\mathbf{U} \cdot \nabla T_{B}\right), \theta\right\rangle+R a\langle\theta, W\rangle, \\
D=\xi\|\nabla \theta\|^{2}+\|\mathbf{U}\|^{2}+D a\|\nabla \mathbf{U}\|^{2} .
\end{gathered}
$$

From equations (16)-(20b), (and the Poincaré inequality $\pi^{2}\|\theta\|^{2} \leq\|\nabla \theta\|^{2}$ ), we can write

$$
\frac{d E}{d t} \leq-2 \pi^{2}(1-m) E
$$

where

$$
m=\max _{H}\left(\frac{I}{D}\right)
$$

and $H=\left\{(\mathbf{U}, \theta) \in L^{2}(V): \nabla \cdot \mathbf{U}=0, \quad W=\theta=0\right.$ at $\left.z= \pm \frac{1}{2}\right\}$. Integrating the above inequality (21), guarantees $E(t) \rightarrow 0$ as $t \rightarrow \infty$ for $0<m<1$. Assuming the critical condition $m=1$ in the maximization problem, the associated Euler-Lagrange equations are

$$
\begin{gathered}
-\xi \nabla T_{B} \theta+R a \theta \hat{\mathbf{k}}-2 m \mathbf{U}+2 m D a \triangle \mathbf{U}=\nabla \lambda, \\
-\xi\left(\mathbf{U} \cdot \nabla T_{B}\right)+R a W+2 m \xi \triangle \theta=0,
\end{gathered}
$$

where $\lambda$ is a Lagrange multiplier which is introduced as $\mathbf{U}$ is solenoidal. Applying the double curl to equation (23a), and taking third component in order to remove $\lambda$, we obtain

$$
\begin{aligned}
& 2\left(\frac{\partial^{2} W}{\partial x^{2}}+\frac{\partial^{2} W}{\partial y^{2}}\right)-R a\left(\frac{\partial^{2} \theta}{\partial x^{2}}+\frac{\partial^{2} \theta}{\partial y^{2}}\right)+\xi \frac{d T_{B}}{d z}\left(\frac{\partial^{2} \theta}{\partial x^{2}}+\frac{\partial^{2} \theta}{\partial y^{2}}\right)-2 D a\left(\frac{\partial^{2}}{\partial x^{2}}+\frac{\partial^{2}}{\partial y^{2}}\right) \triangle W \\
& +2 D a \frac{\partial}{\partial z}\left(\triangle\left(\frac{\partial U}{\partial x}+\frac{\partial V}{\partial y}\right)\right)-2 \frac{\partial}{\partial z}\left(\frac{\partial U}{\partial x}+\frac{\partial V}{\partial y}\right)=0 .
\end{aligned}
$$

Using normal modes, as given in the equation (14), to the equations (23a) and (24), the eigenvalue problem for nonlinear theory is given as

$$
\begin{gathered}
D a\left(D^{2}-a^{2}\right)^{2} W-\left(D^{2}-a^{2}\right) W+\frac{1}{2} \xi \frac{d T_{B}}{d z} a^{2} \theta-\frac{1}{2} a^{2} R a \theta=0, \\
\left(D^{2}-a^{2}\right) \theta-\frac{1}{2} \frac{d T_{B}}{d z} W+\frac{1}{2 \xi} R a W=0, \\
z= \pm \frac{1}{2}: \quad W=D W=\theta=0 .
\end{gathered}
$$

Equations (25a)-(25c) constitutes an eigenvalue problem for $R a$ as a function of $P e, D a$ and $Q$. In addition this eigenvalue problem involves a positive coupling parameter $\xi$ which is to be selected optimally. The procedure to find optimal $\xi$ now follows.

Rescaling the temperature perturbation as

$$
\widetilde{\theta}=\theta \sqrt{\xi}
$$


the modified $I$ and $D$ are

$$
\begin{gathered}
\widetilde{I}=-\sqrt{\xi}\left\langle\left(\mathbf{U} \cdot \nabla T_{B}\right) \widetilde{\theta}\right\rangle+\frac{R a}{\sqrt{\xi}}\langle\widetilde{\theta} W\rangle, \\
\widetilde{D}=\|\nabla \widetilde{\theta}\|^{2}+\|\mathbf{U}\|^{2}+D a\|\nabla \mathbf{U}\|^{2},
\end{gathered}
$$

where we are investigating the maximum of $\frac{\widetilde{I}}{\widetilde{D}}$. To solve this maximization problem, we find the Euler-Lagrange equations are of the form

$$
\begin{gathered}
X \theta \hat{\mathbf{k}}-2 \mathbf{U}+2 D a \triangle \mathbf{U}=p_{, i}, \\
X W+2 \triangle \theta=0 .
\end{gathered}
$$

(where the $\sim$ has been removed for the ease of notation), and

$$
X=\frac{R a}{\sqrt{\xi}}-\frac{d T_{B}}{d z} \sqrt{\xi}
$$

Let $\left(\mathbf{U}^{(1)}, \theta^{(1)}\right)$ and $\left(\mathbf{U}^{(2)}, \theta^{(2)}\right)$ be solutions to above equation corresponding to $\xi^{(1)}$ and $\xi^{(2)}$, respectively. We take the above equation with the superscript (1), multiply each equation with $\mathbf{U}^{(2)}, \theta^{(2)}$ respectively, and integrate over $V$ in each case. The same procedure is repeated with superscripts (1) and (2) reversed. After dividing the system obtained from this procedure by $\xi^{(2)}-\xi^{(1)}$ and taking the limit $\xi^{(2)} \rightarrow \xi^{(1)}$, it follows that

$$
\frac{\partial}{\partial \xi}\langle X W \theta\rangle=0
$$

which implies that the optimal coupling parameter as

$$
\xi=-\frac{R a}{\left(\frac{d T_{B}}{d z}\right)} .
$$

Clearly $\xi>0$ as $\frac{d T_{B}}{d z}<0$. This optimal value of $\xi$ is used in the eigenvalue problem of nonlinear theory (25a)-(25c), in the numerical experiments.

\section{Results and discussion}

The eigenvalue problems (15a)-(15c) for linear theory and (25a)-(25c) for nonlinear theory have been solved numerically using shooting and Runge-Kutta methods. For the shooting method, the boundary value problems (15a)-(15c) and (25a)-(25c), can be reduced to initial value problem with the following conditions

$$
\begin{gathered}
W\left(-\frac{1}{2}\right)=0, \quad D W\left(-\frac{1}{2}\right)=0, \quad D^{2} W\left(-\frac{1}{2}\right)=1, \quad D^{3} W\left(-\frac{1}{2}\right)=\chi \\
\theta\left(-\frac{1}{2}\right)=0, \quad D \theta\left(-\frac{1}{2}\right)=\zeta
\end{gathered}
$$

where $D^{2} W\left(-\frac{1}{2}\right)=1$ is the normalization condition for breaking the scaling invariance of eigenfunctions in (15a)-(15c) and (25a)-(25c). The unknown parameters $\chi, \zeta$, can be evaluated 
by the shooting method with the target condition $W\left(\frac{1}{2}\right)=D W\left(\frac{1}{2}\right)=\theta\left(\frac{1}{2}\right)=0$. More precisely, for fixed values of $Q, P e, D a$, one can find $\chi, \zeta, R a$ in the initial value problems (eigenvalue problem (15a)-(15c), (25a)-(25c) with replacement of initial condition (26)) by using the RungeKutta method. While implementing this procedure, we find eigenvalue $R a(a)$ for a fixed set of flow governing parameters. Rayleigh number $R a$ is minimized over wave number $a$ with the condition $\partial R / \partial a=0$. Critical Rayleigh number $R a_{L}$ (or $R a_{E}$ ) refers to the minimum value of $R a$ at critical wave number $a_{L}$ (or $a_{E}$ ). The critical Rayleigh number for linear and nonlinear theories, are defined as

$$
\begin{gathered}
R a_{L}=\min _{a} R a(a, Q, P e, D a), \\
R a_{E}=\max _{\xi} \min _{a} R a(a, Q, P e, D a, \xi),
\end{gathered}
$$

respectively. To perform the Runge-Kutta method, a built in function, ode45 was used in the software package Matlab R2012b. The critical Rayleigh numbers for both the linear and nonlinear theories have been compared in this section for a fixed set governing parameters $Q, P e$, and $D a$. Throughout the discussion, the solid lines indicate neutral stability curves for linear instability thresholds, whereas dashed lines indicate neutral stability curves for nonlinear stability thresholds. In this article, $P e>0$ refers to upward throughflow whereas $P e<0$ refers to downward throughflow.

The Darcy flow model can be recovered by putting $D a=0$ in the equations (15a) and (25a). Small values of the Darcy number $D a$ cause system of differential equations to become stiff and creates numerical difficulties. Therefore, the range of the Darcy number is restricted to $10^{-3} \leq D a \leq 10^{2}$ during the numerical computations. Higher values of the internal heat source $Q$ causes convective instability even in the absence of external temperature gradient. Thus, the neutral stability curves are presented for $0 \leq Q \leq 50$ and $-20 \leq P e \leq 20$.

In the absence of vertical throughflow and the Brinkman term, the present problem reduces to the study by Gasser and Kazimi [12]. Table 1 shows a very good agreement of the present numerical results with the external Rayleigh number $\tilde{R_{E}}$ given in Gasser and Kazimi [12]. In both the linear and nonlinear theories critical Rayleigh numbers $R a_{L}$ and $R a_{E}$ increase for upward throughflow, and decrease for downward throughflow. This observation is consistent with the observation made by Nield and Kuznetsov [27].

When $Q=0$ and $D a=0$, the present problem can be reduced to the study of in Barletta et al. [31]. From Table 2, the comparison between Barletta et al. [31] and the present linear stability theory results $(Q=0$ and $D a=0)$ demonstrate excellent agreement.

Fig. 1 represents neutral stability curves for different governing parameters. Fig.1a shows that in the absence of throughflow, both linear and nonlinear thresholds coincide. This observation is consistent with the classical Darcy-Bénard convection in porous media as reported in Straughan [32]. These two theories give different stability thresholds as soon as the throughflow is introduced in the medium $(P e \neq 0)$. It is observed that increasing the value of $P e$, increases the critical thermal Rayleigh number in both linear and nonlinear theories. Fig.1b shows that $R a$ increases as the Darcy number increases. Fig. 2 displays neutral curves for the onset of convection for $Q=0,2$ where $D a=0.01$ and $P e=-5,5$. In the case of downward throughflow $(P e=-5)$, $R a$ decreases as $Q$ increases for certain range of wave number. However, in the case of upward 
Table 1: Comparison between the linear stability results of Gasser and Kazimi [12] and this article's linear stability results at $P e=0$.

\begin{tabular}{|c|l|l|}
\hline$Q$ & $\begin{array}{l}\text { Gasser and Kazimi }[12] \\
\tilde{R_{E}}\end{array}$ & $R a_{L}$ \\
\hline 0 & 39.48 & 39.4783 \\
5 & 34.59 & 34.5950 \\
10 & 27.02 & 27.0160 \\
15 & 21.45 & 21.4461 \\
20 & 17.63 & 17.6264 \\
25 & 14.92 & 14.9163 \\
30 & 12.91 & 12.9115 \\
40 & 10.16 & 10.1605 \\
50 & 8.37 & 8.3689 \\
60 & 7.11 & 7.1120 \\
80 & 5.47 & 5.4669 \\
100 & 4.44 & 4.4390 \\
\hline
\end{tabular}

Table 2: Comparison between the linear stability results of Barletta et al. [31] (for the case of Gebhart number $G e=0$ ) and this article's linear stability results for $Q=0$ and $D a=0$.

\begin{tabular}{|c|l|l|}
\hline$P e$ & $\begin{array}{l}\text { Barletta et al. [31] } \\
\text { Critical value of } R a\end{array}$ & $R a_{L}$ \\
\hline-15 & 215.283 & 215.2828 \\
-10 & 143.518 & 143.5185 \\
-5 & 73.4146 & 73.41465 \\
-0.001 & 39.4784 & 39.47839 \\
0.001 & 39.4784 & 39.47839 \\
5 & 73.4146 & 73.4144 \\
10 & 143.518 & 143.5182 \\
15 & 215.283 & 215.2824 \\
\hline
\end{tabular}




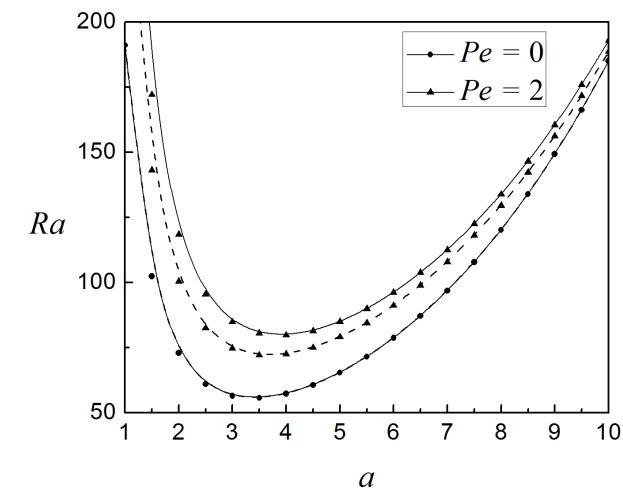

(a) $Q=5, D a=0.01$

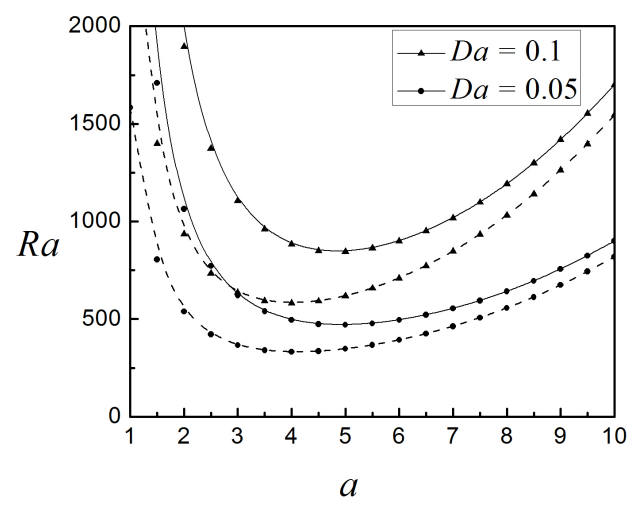

(b) $Q=5, P e=5$

Figure 1: Neutral stability curves for the onset of convection. Solid lines represent linear theory and dashed lines represent nonlinear theory.

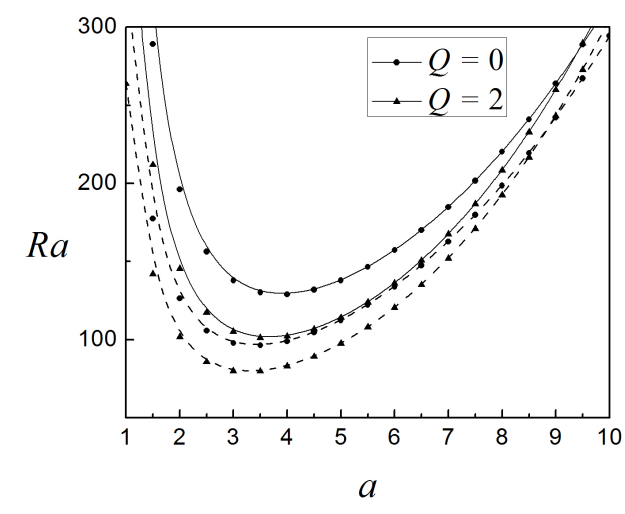

(a) $P e=-5$

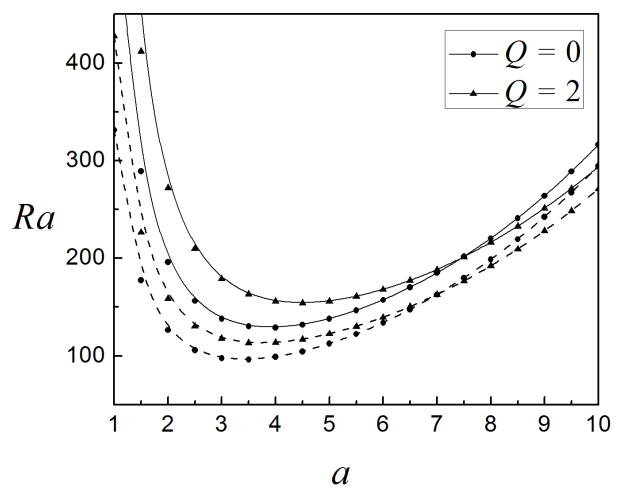

(b) $P e=5$

Figure 2: Neutral stability curves for $D a=0.01$. Solid lines represent linear theory and dashed lines represent nonlinear theory.

throughflow $(P e=5), R a$ increases as $Q$ increases. This demonstrates that upward throughflow delays the onset of convection even in the presence of internal heat source.

The critical thermal Rayleigh number plotted against the internal heat source parameter is displayed in fig. 3 for the values of $D a=0.1$ and $P e=5,-5$. In fig. 3a it can be observed that $R a_{L}$ and $R a_{E}$ decrease with an increase in the value of the internal heat source parameter. Since, the internal heat source raises the global temperature of the medium and causes instability to commence. Furthermore, it is observed that the stability thresholds for nonlinear theory are smaller than the instability thresholds for the linear theory, clearly leading to sub-critical instabilities in the medium. For smaller values of the internal heat source parameter $\mathrm{Q}$, the region of sub-critical instabilities is large. This sub-critical region of instabilities is narrowed with increasing internal heat source in the medium. Fig. 3b shows that (for the range of values considered for $Q)$ the results obtained for the upward throughflow $(P e=5)$ show the opposite 


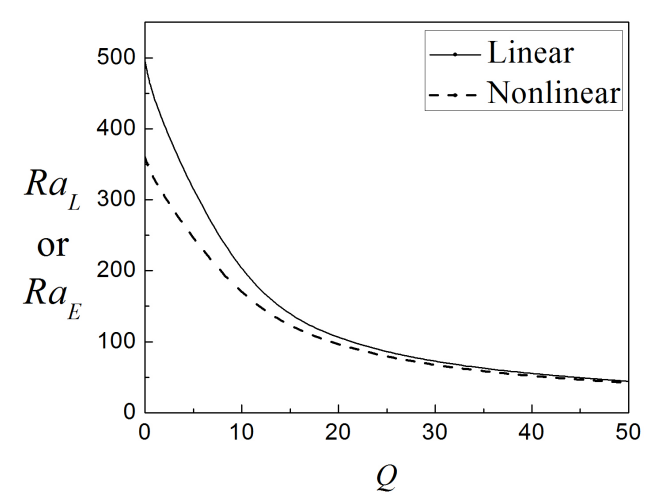

(a) $P e=-5$

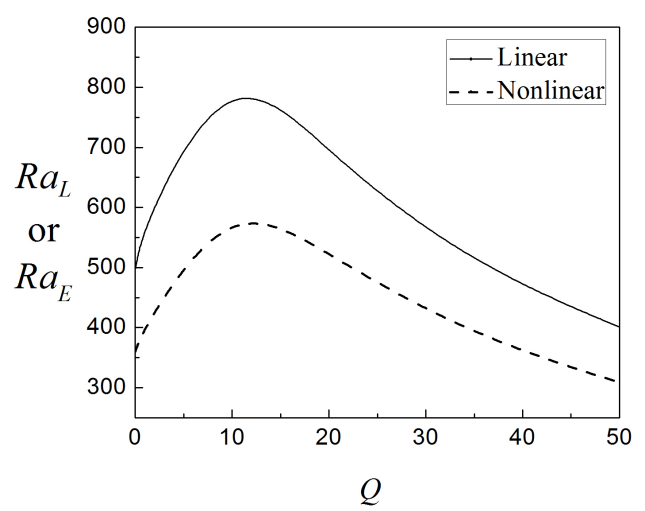

(b) $\mathrm{Pe}=5$

Figure 3: Variation of $R a_{L}$ and $R a_{E}$ against $Q$ for $D a=0.1$.

trend to the results obtained for the downward throughflow $(P e=-5)$. For $P e=5$, the upward throughflow dominates the internal heating effect and causes the stabilization of the flow. From fig. 3b, it is observed that upward throughflow dominates nearly up to $Q=10$ and for higher values of $Q=10$, the internal heating effect becomes significant to cause destabilization.

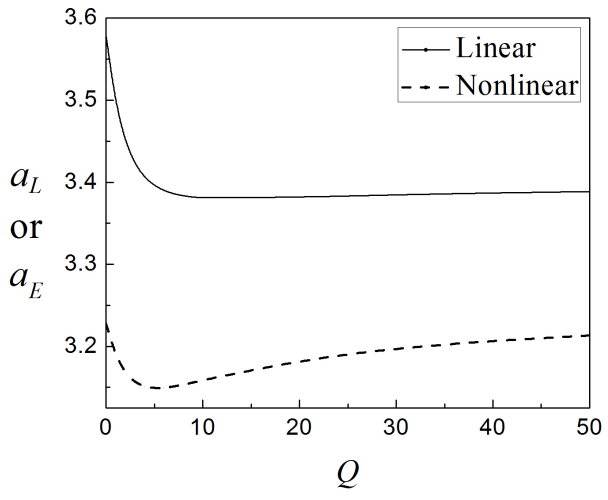

(a) $P e=-5$

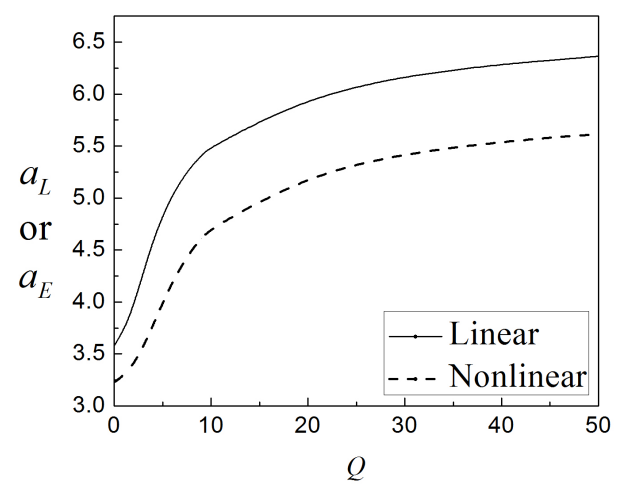

(b) $P e=5$

Figure 4: Variation of critical wave number $a_{L}$ and $a_{E}$ with $Q$ for $D a=0.1$.

Fig. 4 exhibits the variation of critical wave number $a_{L}$ (and $a_{E}$ ) versus $Q$ for the same set of parameters used in fig. 3. For $P e=-5$ (downward throughflow), the critical wavenumber decreases with an increasing value of the internal heat source parameter $Q=6$, beyond $Q=6$ the critical wavenumber increases with $Q$. While for $P e=5$ (upward throughflow), the critical wavenumber increases with $Q$.

Fig. 5 presents the response of $R a_{L}$ (or $R a_{E}$ ) to vertical throughflow $P e$ for $Q=1,5$ and $D a=0.1$. In the figures $5 \mathrm{a}$ and $5 \mathrm{~b}$, the critical thermal Rayleigh numbers $R a_{L}$ and $R a_{E}$ are increasing with the magnitude of $P e$. When $P e=0$, the linear and nonlinear stability theories show good agreement. As $Q$ increases, the flow is destabilized for downward throughflow, and stabilization is seen as the $P e$ dominates $Q$ in the upward throughflow. The region of sub-critical 


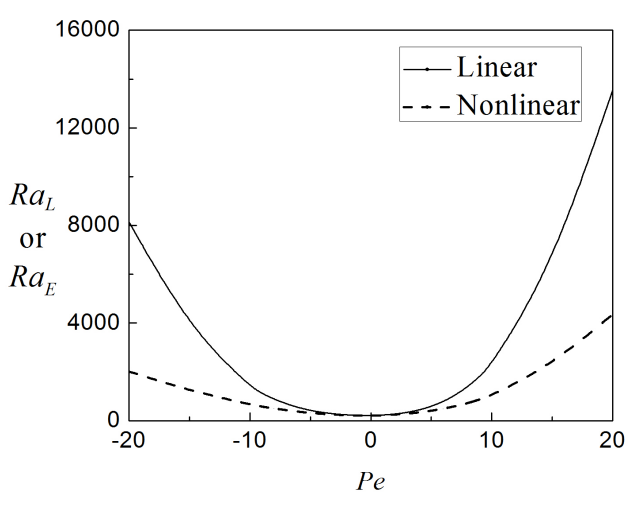

(a) $Q=1$

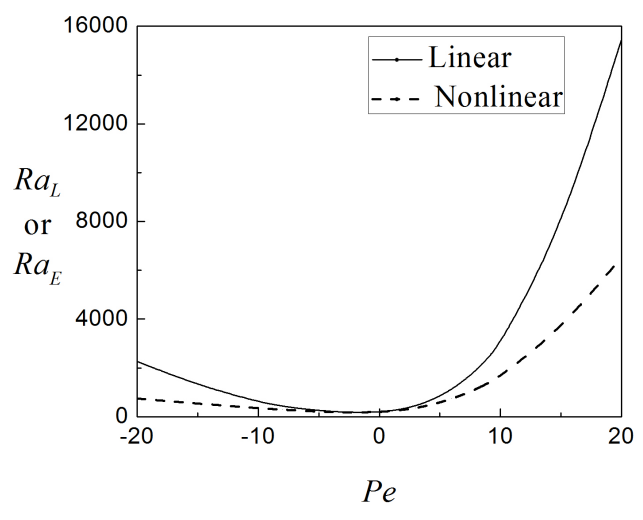

(b) $Q=5$

Figure 5: Variation of $R a_{L}$ and $R a_{E}$ against $P e$ for $D a=0.1$.

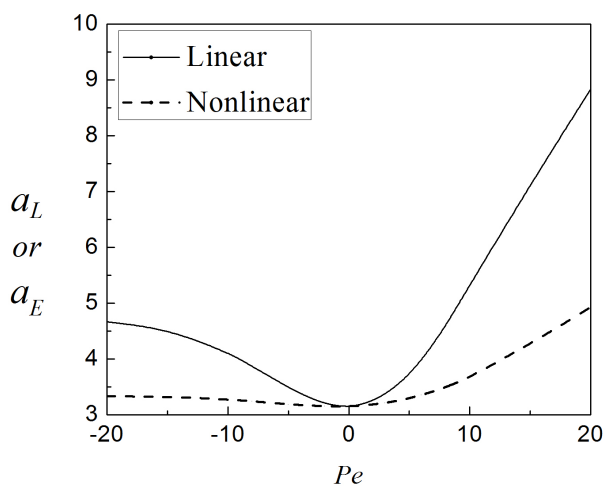

(a) $Q=1$

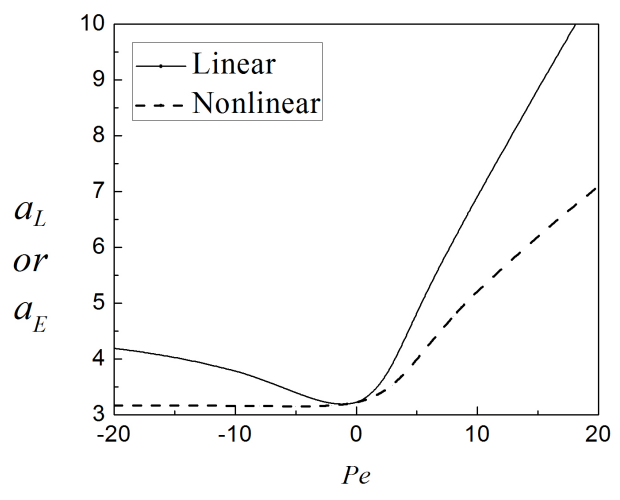

(b) $Q=5$

Figure 6: Variation of critical wave number $a_{L}$ and $a_{E}$ with $P e$ for $D a=0.1$.

instabilities increases with the magnitude of $P e$. The variation of $a_{L}$ (or $a_{E}$ ) against $P e$ is shown in fig. 6 , for the same set of other parameters used in fig. 5 .

Fig. 7a depicts the linear and nonlinear stability curves for $Q=5, P e=5$ with varying of Darcy number $D a$. Small values of Darcy number $D a$ has no significant impact on $R a_{L}$ and $R_{E}$, but when Darcy number $D a$ increased beyond 0.1, it showed considerable stabilizing effect. The region of sub-critical instabilities increases with the Darcy number. From fig. 7b, it is observed that $a_{L}$ and $a_{E}$ are decreasing with increasing $D a$.

\section{Conclusions}

The onset of the instability of a vertical throughflow with a uniform internal heat source in a porous medium has been studied, where the flow is governed by the Brinkman-extended Darcy law. Linear and nonlinear stability analyses have been conducted, where the nonlinear stability theory was performed by using Energy Method. The eigenvalue problem in both the linear and nonlinear theories was solved numerically using shooting and Runge-Kutta methods. The critical 


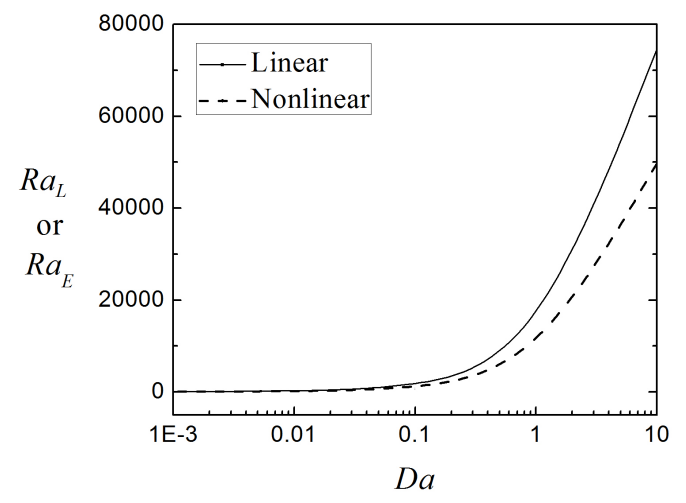

(a) $R a_{L}\left(\right.$ or $\left.R a_{E}\right)$ versus $D a$

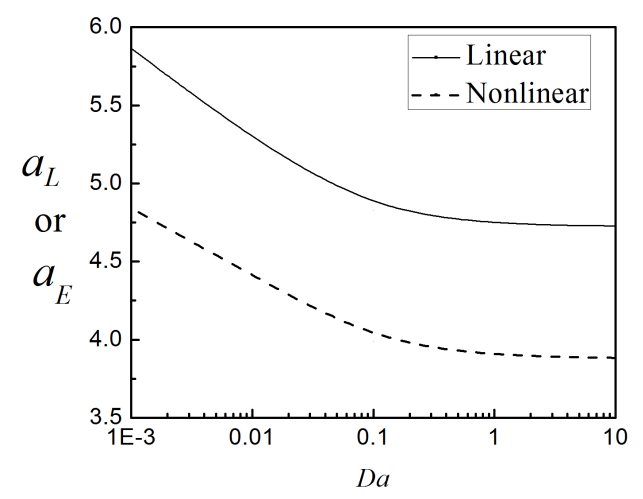

(b) $a_{C}\left(\right.$ or $\left.a_{E}\right)$ versus $D a$

Figure 7: Plots of $R a_{L}$ (or $R a_{E}$ ) and $a_{L}$ (or $a_{E}$ ) against $D a$ for $Q=5, P e=5$.

Rayleigh number for the linear and nonlinear theories were compared for Darcy number, Péclet number and internal heat source coefficient. The main observations from the obtained results are:

- When the upward throughflow is strong enough $(P e=5)$, the effect of throughflow dominates the effect of internal heat source parameter (for $Q=1$ to 7 ) at the onset of convection.

- In the absence of throughflow, the linear and nonlinear theories give the same result.

- Lower values of Darcy number have insignificant stabilization effect comparing to the values of Darcy number greater than 0.1.

\section{References}

[1] C. W. Horton, F. T. Rogers, Convection currents in a porous medium, Journal of Applied Physics 16 (6) (1945) 367-370.

[2] E. R. Lapwood, Convection of a fluid in a porous medium 44 (04) (1948) 508-521.

[3] H. C. Brinkman, A calculation of the viscous force exerted by a flowing fluid on a dense swarm of particles, Applied Scientific Research 1 (1) (1949) 27-34.

[4] C. K. W. Tam, The drag on a cloud of spherical particles in low Reynolds number flow, Journal of Fluid Mechanics 38 (03) (1969) 537-546.

[5] K. Vafai, C. Tien, Boundary and inertia effects on flow and heat transfer in porous media, International Journal of Heat and Mass Transfer 24 (2) (1981) 195-203.

[6] D. A. Nield, The boundary correction for the Rayleigh-Darcy problem: limitations of the brinkman equation, Journal of Fluid Mechanics 128 (1983) 37-46.

[7] K. Vafai, S. J. Kim, On the limitations of the Brinkman-Forchheimer-extended Darcy equation, International Journal of Heat and Fluid Flow 16 (1) (1995) 11-15. 
[8] D. A. Nield, The limitations of the Brinkman-Forchheimer equation in modeling flow in a saturated porous medium and at an interface, International Journal of Heat and Fluid Flow 12 (3) (1991) 269-272.

[9] K. Walker, G. M. Homsy, A note on convective instabilities in Boussinesq fluids and porous media, A. S. M. E .Journal of Heat Transfer 99 (1977) 338-339.

[10] D. A. S. Rees, The onset of Darcy-Brinkman convection in a porous layer: an asymptotic analysis, International Journal of Heat and Mass Transfer 45 (11) (2002) 2213-2220.

[11] A. Barletta, E. R. di Schio, M. Celli, Instability and viscous dissipation in the horizontal Brinkman flow through a porous medium, Transport in Porous Media 87 (1) (2011) 105-119.

[12] R. D. Gasser, M. S. Kazimi, Onset of convection in a porous medium with internal heat generation, Journal of Heat Transfer 98 (1) (1976) 49-54.

[13] M. Tveitereid, Thermal convection in a horizontal porous layer with internal heat sources, International Journal of Heat and Mass Transfer 20 (10) (1977) 1045-1050.

[14] J. J. Royer, L. Flores, Two-dimensional natural convection in an anisotropic and heterogeneous porous medium with internal heat generation, International journal of Heat and Mass transfer 37 (9) (1994) 1387-1399.

[15] C. Parthiban, P. R. Patil, Thermal instability in an anisotropic porous medium with internal heat source and inclined temperature gradient, International communications in heat and mass transfer 24 (7) (1997) 1049-1058.

[16] A. Nouri-Borujerdi, A. R. Noghrehabadi, D. A. S. Rees, Onset of convection in a horizontal porous channel with uniform heat generation using a thermal nonequilibrium model, Transport in porous media 69 (3) (2007) 343-357.

[17] A. Nouri-Borujerdi, A. R. Noghrehabadi, D. A. S. Rees, Influence of Darcy number on the onset of convection in a porous layer with a uniform heat source, International Journal of Thermal Sciences 47 (8) (2008) 1020-1025.

[18] R. A. Wooding, Rayleigh instability of a thermal boundary layer in flow through a porous medium, Journal of fluid mechanics 9 (02) (1960) 183-192.

[19] F. M. Sutton, Onset of convection in a porous channel with net through flow, Physics of Fluids (1958-1988) 13 (8) (1970) 1931-1934.

[20] G. M. Homsy, A. E. Sherwood, Convective instabilities in porous media with through flow, AIChE Journal 22 (1) (1976) 168-174.

[21] D. A. Nield, Convection in a porous medium with inclined temperature gradient and vertical throughflow, International journal of heat and mass transfer 41 (1) (1998) 241-243. 
[22] Z. Qiao, P. N. Kaloni, Non-linear convection in a porous medium with inclined temperature gradient and vertical throughflow, International journal of heat and mass transfer 41 (16) (1998) 2549-2552.

[23] A. Khalili, I. Shivakumara, Onset of convection in a porous layer with net through-flow and internal heat generation, Physics of Fluids (1994-present) 10 (1) (1998) 315-317.

[24] C. J. V. Duijn, G. J. M. Pieters, R. A. Wooding, A. V. D. Ploeg, Stability criteria for the vertical boundary layer formed by throughflow near the surface of a porous medium, Environmental Mechanics: Water, Mass and Energy Transfer in the Biosphere: The Philip Volume (2002) 155-169.

[25] G. J. M. Pieters, H. M. Schuttelaars, On the nonlinear dynamics of a saline boundary layer formed by throughflow near the surface of a porous medium, Physica D: Nonlinear Phenomena 237 (23) (2008) 3075-3088.

[26] P. M. Patil, D. A. S. Rees, Linear instability of a horizontal thermal boundary layer formed by vertical throughflow in a porous medium: the effect of local thermal nonequilibrium, Transport in porous media 99 (2) (2013) 207-227.

[27] A. V. Kuznetsov, D. A. Nield, The effect of vertical throughflow on the onset of convection induced by internal heating in a layered porous medium, Transport in porous media 100 (1) (2013) 101-114.

[28] A. V. Kuznetsov, D. A. Nield, Local thermal non-equilibrium effects on the onset of convection in an internally heated layered porous medium with vertical throughflow, International Journal of Thermal Sciences 92 (2015) 97-105.

[29] N. Deepika, P. A. L. Narayana, Effects of vertical throughflow and variable gravity on Hadley-Prats flow in a porous medium, Transport in Porous Media 109 (2) (2015) 455-468.

[30] A. Barletta, L. Storesletten, Linear instability of the vertical throughflow in a horizontal porous layer saturated by a power-law fluid, International Journal of Heat and Mass Transfer 99 (2016) 293-302.

[31] A. Barletta, E. R. di Schio, L. Storesletten, Convective roll instabilities of vertical throughflow with viscous dissipation in a horizontal porous layer, Transport in porous media 81 (3) (2010) 461-477.

[32] B. Straughan, The Energy Method, Stability, and Nonlinear Convection, 2nd Edition, Springer-Verlag New York, 2004. 